\title{
Non-commutativity from the double sigma model
}

\author{
Dimitri Polyakov, Peng Wang, Houwen Wu and Haitang Yang \\ Center for theoretical physics, Sichuan University, \\ Chengdu, 610064, China \\ E-mail: polyakov@scu.edu.cn, pengw@scu.edu.cn, iverwu@stu.scu.edu.cn, \\ hyanga@scu.edu.cn
}

AbSTRACT: We show how non-commutativity arises from commutativity in the double sigma model. We demonstrate that this model is intrinsically non-commutative by calculating the propagators. In the simplest phase configuration, there are two dual copies of commutative theories. In general rotated frames, one gets a non-commutative theory and a commutative partner. Thus a non-vanishing $B$ also leads to a commutative theory. Our results imply that $O(D, D)$ symmetry unifies not only the big and small torus physics, but also the commutative and non-commutative theories. The physical interpretations of the metric and other parameters in the double sigma model are completely dictated by the boundary conditions. The open-closed relation is also an $O(D, D)$ rotation and naturally leads to the Seiberg-Witten map. Moreover, after applying a second dual rotation, we identify the description parameter in the Seiberg-Witten map as an $O(D, D)$ group parameter and all theories are non-commutative under this composite rotation. As a bonus, the propagators of general frames in double sigma model for open string are also presented.

Keywords: String Duality, Bosonic Strings

ArXiv EPrint: 1501.01550v2

Open Access, (C) The Authors.

Article funded by $\mathrm{SCOAP}^{3}$. 


\section{Contents}

2 Non-commutativity in string theory and the Seiberg-Witten map

$3 O(D, D)$ rotations of the propagators

\section{Introduction}

Symmetries and dualities play a central role in modern physics. There are some evidences that various dualities in string theory may emerge from symmetry breaking in some select backgrounds. In string theory, there are three well known dualities: T-duality relates large and small distance physics; S-duality connects weak and strong couplings; and Uduality unifies the T-duality and S-duality. M-theory is dictated by these three types of dualities. There is another significant equivalence in string theory, the Seiberg-Witten map [1] which seems to disconnect from known dualities or symmetries. The SeibergWitten map relies on the non-commutativity from string theory and the open-closed relation. In the language of string theory, commutativity is a natural description for closed string and non-commutativity is natural for open string. The Seiberg-Witten map is a map between commutative variables $\left(\hat{g}_{s}, \hat{g}_{i j}, \hat{B}_{i j}\right)$ of closed strings and non-commutative variables $\left(g_{s}, g_{i j}, B_{i j}\right)$ of open strings. The Dirac-Born-Infeld (DBI) action, obtained from calculating the one-loop beta function of worldsheet action, is originally expressed with commutative variables $\left(\hat{g}_{s}, \hat{g}_{i j}, \hat{B}_{i j}\right)$. Utilizing the Seiberg-Witten map, one can derive a non-commutative version of the DBI action. Therefore, the Seiberg-Witten map unifies the non-commutative gauge theories and commutative gauge theories.

In this work, we present solid evidences to show that the Seiberg-Witten map can be described by a pure coordinate transformation of $O(D, D)$ group. The relationship between non-commutative property and T-duality was first proposed by Connes, Douglas and Schwarz [2], where they showed that the Matrix model on tori with an anti-symmetric field produces non-commutativity in a natural way. Non-commutativities from open string ending on D-branes are first addressed in $[3,4]$. In [5], Maharana and Pal made a nice try to construct a relationship between $O(D, D)$ transformations and the Seiberg-Witten map. They introduced T-dual coordinates in an ad hoc way and found some links between $O(D, D)$ and the Seiberg-Witten map. But since their setup is not a consistent $O(D, D)$ covariant theory, the underlying connection of $O(D, D)$ and the Seiberg-Witten map was not revealed. 
The $O(D, D)$ symmetry is a continuous symmetry for non-compact background, where $D$ is a number of spacetime dimensions. If we compactify $d=D-n$ dimensions, the continuous $O(D, D)$ breaks into an $O(n, n) \times O(d, d ; \mathbb{Z})$ group. The $O(d, d ; \mathbb{Z})$ group is known as T-duality group in the compactified background. Moreover, it is well known that the solution space of the closed string low energy effective action possesses $O(n, n)$ symmetry for certain backgrounds. These features makes it tempting to construct theories having $O(D, D)$ invariance at the very beginning. The double sigma model was proposed by Tseytlin $[6,7]$ and developed in $[8,9]$ to fulfill this purpose. Recent developments of double field theory [10-23] have the similar inspirations. In the double sigma action, the $O(D, D)$ invariance is manifested by introducing another set of target space coordinates. In the same pattern of Polyakov action, open string and closed string share the same double sigma action. Whether the theory represents open or closed string is determined by the boundary conditions. In this paper, we will address the open string scenario. Surprisingly, after calculating the propagators, we find that the double sigma model intrinsically has both commutativity and non-commutativity. Starting from the simplest phase configuration, there are two commutative theories, either of which can represent our ordinary picture. However, the hidden mixed propagators between the dual theories exhibit noncommutative property, which implies that the ordinary coordinate $X$ is non-commutative to its dual $\tilde{X}$. With this property, it is not hard to see that after an $O(D, D)$ rotation, a non-commutative theory emerges. Our calculation shows that this non-commutative theory is completely identical to that in $[1,24-26]$. It turns out in general phase frame, besides the non-commutative theory, we have another commutative companion even if the noncommutative parameter is nonvanishing. The Neven-Schwartz $B$ field is simply a group parameter in this theory. Another very interesting observation is that, unlike the situation in the Polyakov action, the physical interpretations of the metric and other parameters in the double sigma action are completely dictated by the boundary conditions. The parameters between closed and open strings are linked by an $O(D, D)$ transformation. With all these results in mind, we conclude that the double sigma action unifies both commutativity and non-commutativity and Seiberg-Witten map can be described by a subset of $O(D, D)$ group. It is quite inspiring to notice that $O(D, D)$ group not only includes dual theories of small and large tori, but also unifies the commutative and non-commutative theories. The unification of the commutative and non-commutative theories is realized by the intrinsic non-commutative property of the action, observation of commutativity or non-commutativity depends on which frame is taken.

It is remarkable that after performing another dual rotation with a group parameter $C$, we find that the description parameter $\Phi$ in the Seiberg-Witten map is not necessary any more and can be removed. For fixed closed string parameters, the corresponding open string parameters are not unique. The arbitrariness of the non-commutative parameter $\theta$ is accounted by the the alterable open string metric $g$, which in turn derived from the arbitrariness of the group parameter $C$. Therefore, $C$ replaces the role of $\Phi$ to make the non-commutative theory is optional for different metric and $*$ product parameter $\theta$. Another feature of the composite rotation is that both the ordinary theory and its dual become non-commutative. 
The reminder of this paper is outlined as follows. In section 2, we briefly review the non-commutativity in string theory and the Seiberg-Witten map. By using the double sigma model, we derive the non-commutative theory from a commutative one through $O(D, D)$ coordinate transformations in section 3. We address the general description under a composite rotation in section 4 .

\section{Non-commutativity in string theory and the Seiberg-Witten map}

This short review is based on Seiberg and Witten's work [1], and Mukhi's talk [27]. We begin with Euclidean worldsheet action

$$
S=\frac{1}{2} \int_{\Sigma}\left(g_{i j} \partial_{\alpha} X^{i} \partial^{\alpha} X^{j}-i \epsilon^{\alpha \beta} B_{i j} \partial_{\alpha} X^{i} \partial_{\beta} X^{j}\right)
$$

where $\epsilon$ is an anti-symmetric matrix, $g$ is the spacetime metric. The worldsheet equation of motion is given by

$$
g_{i j} \partial_{\alpha} \partial^{\alpha} X^{j}=0
$$

The open string boundary condition is

$$
g_{i j} \partial_{\sigma} X^{j}+\left.i B_{i j} \partial_{\tau} X^{i}\right|_{\partial \Sigma}=0
$$

To calculate the correlation function, we map the disc to the upper half $z$ plane by $z=$ $\tau+i \sigma$. The propagator is

$$
\begin{aligned}
\left\langle X^{i}(z, \bar{z}) X^{j}\left(z^{\prime}, \bar{z}^{\prime}\right)\right\rangle= & -\alpha^{\prime}\left[g^{i j} \log \left|z-z^{\prime}\right|-g^{i j} \log \left|z-\bar{z}^{\prime}\right|\right. \\
& \left.+G^{i j} \log \left|z-\bar{z}^{\prime}\right|^{2}+\frac{1}{2 \pi \alpha^{\prime}} \theta^{i j} \log \frac{z-\bar{z}^{\prime}}{\bar{z}-z^{\prime}}+D^{i j}\right],
\end{aligned}
$$

where

$$
G_{i j}=\left(g-B g^{-1} B\right)_{i j}, \quad \theta^{i j}=-\left(\frac{1}{g-B} B \frac{1}{g+B}\right)^{i j}
$$

and can be grouped as

$$
\frac{1}{G}+\theta=\frac{1}{g+B}
$$

Now, consider the propagator on the boundary and one gets

$$
\left\langle X^{i}(\tau) X^{j}\left(\tau^{\prime}\right)\right\rangle=-\alpha^{\prime}\left[2 G^{i j} \log \left|\tau-\tau^{\prime}\right|+\frac{i}{2} \theta^{i j} \varepsilon\left(\tau-\tau^{\prime}\right)\right],
$$

where $\varepsilon\left(\tau-\tau^{\prime}\right)=+1$ when $\tau-\tau^{\prime}>0$, and $\varepsilon\left(\tau-\tau^{\prime}\right)=-1$ when $\tau-\tau^{\prime}<0$. Then, $G^{i j}$ is effectively identified as the open string metric. The antisymmetric quantity $\theta^{i j}$ introduces non-commutativity into the theory. To see the map between commutative and non-commutative gauge theories, we turn to the DBI action

$$
S_{\mathrm{DBI}}=\frac{1}{g_{s}} \int d^{D} x \sqrt{\operatorname{det}(g+B+F)}
$$


where $g_{s}$ is the closed string coupling and $F_{i j}=\partial_{i} A_{j}-\partial_{j} A_{i}$ is the gauge field strength. For simplicity, the field strength $F$ is assumed to be constant. Expanding this action in order of $F$, one gets the Maxwell equation where $S \sim \int F_{i j} F^{i j}$. Applying the open-closed string relation between the closed string side $(g, B)$ and the open string side $(G, \theta)$, the DBI action can be rewritten in the terms of $(G, \theta)$ as

$$
S_{\mathrm{DBI}}=\frac{1}{g_{s}} \int d^{D} x \frac{\sqrt{\operatorname{det}(1+\theta F)}}{\sqrt{\operatorname{det}(1+\theta G)}} \sqrt{\operatorname{det}\left(G+F \frac{1}{1+\theta F}\right)} .
$$

After redefining

$$
\hat{F} \equiv F \frac{1}{1+\theta F}, \quad G_{s} \equiv g_{s} \sqrt{\operatorname{det}(1+\theta G)} .
$$

the DBI action becomes

$$
S_{\mathrm{DBI}}=\frac{1}{G_{s}} \int d^{D} x \frac{1}{\sqrt{\operatorname{det}(1-\theta \hat{F})}} \sqrt{\operatorname{det}(G+\hat{F})} .
$$

The coupling $G_{s}$ can be seen as a new string coupling, and $B$ field disappeared. Since the prefactor $\sqrt{\operatorname{det}(1-\theta \hat{F})}$ can be canceled by open Wilson line or understood as a nontrivial Jacobian factor from a local coordinate transformation eliminating $U(1)$ gauge fields [30-32] and does not affect our discussion, the action is simplified as

$$
S_{\mathrm{DBI}}=\frac{1}{G_{s}} \int d^{D} x \sqrt{\operatorname{det}(G+\hat{F})} .
$$

To understand the physical implication of $\hat{F}$, we expand it in order of $\theta$,

$$
\hat{F}_{i j}=F_{i j}-F_{i k} \theta^{k l} F_{l j}+\mathcal{O}\left(\theta^{2}\right) .
$$

Since $F_{i j}=\partial_{i} A_{j}-\partial_{j} A_{i}$, if we redefine a new gauge potential $\hat{A}_{i}$ as

$$
\hat{A}_{i} \equiv A_{i}-\theta^{k l}\left(A_{k} \partial_{l} A_{i}+\frac{1}{2} A_{k} \partial_{i} A_{l}\right)+\mathcal{O}\left(\theta^{2}\right) .
$$

The $\hat{F}$ can be rewritten as

$$
\begin{aligned}
\hat{F}_{i j} & =\partial_{i} \hat{A}_{j}-\partial_{j} \hat{A}_{i}+\theta^{k l} \partial_{k} \hat{A}_{i} \partial_{l} \hat{A}_{j} \\
& =\partial_{i} \hat{A}_{j}-\partial_{j} \hat{A}_{i}+\left\{\hat{A}_{i}, \hat{A}_{j}\right\},
\end{aligned}
$$

where the Poisson bracket $\left\{\hat{A}_{i}, \hat{A}_{j}\right\}$ can be lifted to a non-commutative commutator as $-i\left[\hat{A}_{i}, \hat{A}_{j}\right]_{*}$ through the Moyal-Weyl product as follows

$$
\{f, g\} \rightarrow-i(f * g-g * f),
$$

with

$$
f(x) * g(x)=\left.e^{\frac{i}{2} \theta^{i j} \frac{\partial}{\partial x^{i}} \frac{\partial}{\partial y^{j}}} f(x) g(y)\right|_{x=y}
$$


Therefore, we have two copies of DBI actions, one is commutative

$$
S_{\mathrm{DBI}}=\frac{1}{g_{s}} \int d^{D} x \sqrt{\operatorname{det}(g+B+F)},
$$

the other is non-commutative

$$
S_{\mathrm{DBI}}=\frac{1}{G_{s}} \int d^{D} x \sqrt{\operatorname{det}(G+\hat{F})}
$$

where $\hat{F}_{i j}=\partial_{i} \hat{A}_{j}-\partial_{j} \hat{A}_{i}-i\left[\hat{A}_{i}, \hat{A}_{j}\right]_{*}$. The relations between these two DBI actions, or relations between commutative gauge theories and non-commutative gauge theories are

$$
\begin{aligned}
\hat{F} & =F \frac{1}{1+\theta F}, \\
\hat{A}_{i} & =A_{i}-\theta^{k l}\left(A_{k} \partial_{l} A_{i}+\frac{1}{2} A_{k} \partial_{i} A_{l}\right)+\mathcal{O}\left(\theta^{2}\right) .
\end{aligned}
$$

\section{$3 O(D, D)$ rotations of the propagators}

To understand how the non-commutativity arises from $O(D, D)$ symmetry, we start from the simplest phase configuration of the double sigma model. The action with Lorentz signature is given as follows

$$
S=-\frac{1}{4 \pi \alpha^{\prime}} \int_{\Sigma}\left(-\partial_{1} X^{M} h_{M N} \partial_{1} X^{N}+\partial_{1} X^{M} \eta_{M N} \partial_{0} X^{N}\right)
$$

where $\partial_{0}=\partial_{\tau}, \partial_{1}=\partial_{\sigma}$ and

$$
h_{M N}=\left(\begin{array}{cc}
g_{i j} & 0 \\
0 & g^{i j}
\end{array}\right), \quad \eta_{M N}=\left(\begin{array}{ll}
0 & 1 \\
1 & 0
\end{array}\right), \quad X^{M}=\left(\begin{array}{c}
X^{i} \\
\tilde{X}_{i}
\end{array}\right) .
$$

In the double sigma action, the $D$ dimensional target space coordinate $X^{i}$ is doubled to $2 D$ dimensional $X^{M}\left(X^{i}, \tilde{X}_{i}\right)$, where $M=1,2, \ldots, 2 D$, while the worldsheet $\Sigma$ is still two dimentional. Therefore, $h_{M N}$ and $\eta_{M N}$ are $2 D \times 2 D$ matrices. $g_{i j}$ is the spacetime metric. It is important that this action has an $O(D, D)$ symmetry: invariant under rotation $\Omega$ satisfying $\Omega \eta \Omega^{T}=\eta$. Here for later convenience, we use $X^{M}=\left(X^{i}, \tilde{X}_{i}\right)$ in the action, different from the conventions in $[6,7]$. In this paper, we assume the open string ending on spacefilling branes. The case for $D p$-branes can be easily generalized. It is worth noting that open and closed strings share the same action. The differences between open and closed strings are determined by the boundary conditions. In recent works, the double field theory action of closed strings has been derived from this action [17-23]. The equation of motion (EOM) is obtained by varying $X^{M}$

$$
\partial_{1}\left(h_{M N} \partial_{1} X^{N}-\eta_{M N} \partial_{0} X^{N}\right)=0
$$

which leads to

$$
g \partial_{1} X-\partial_{0} \tilde{X}=f_{1}(\tau)
$$




$$
g^{-1} \partial_{1} \tilde{X}-\partial_{0} X=f_{2}(\tau)
$$

where $f_{1}(\tau)$ and $f_{2}(\tau)$ are arbitrary regular functions solely depending on $\tau$. It turns out that after imposing the self-duality condition $f_{i}=0$, the action (3.1) recedes back to the Polyakov action [6, 7]. This self-duality condition corresponds to the strong constraint in the framework of double field theory [28, 29]. But we will momentarily keep it unfixed and show that, for open strings, the self-duality condition is a derived consequence under $O(D, D)$ covariant boundary condition but not a premise. The EOM (3.3) can be put into decoupled form

$$
\begin{aligned}
& \left(\partial_{1}^{2}-\partial_{0}^{2}\right) X=\partial_{0} f_{2}(\tau) \\
& \left(\partial_{1}^{2}-\partial_{0}^{2}\right) \tilde{X}=\partial_{0} f_{1}(\tau)
\end{aligned}
$$

The boundary conditions of open strings are determined by the EOM

$$
\left.\delta X^{M}\left(h_{M N} \partial_{1} X^{N}-\frac{1}{2} \eta_{M N} \partial_{0} X^{N}\right)\right|_{\partial \Sigma}=0
$$

which can be expanded as

$$
\delta X\left(g \partial_{1} X-\frac{1}{2} \partial_{0} \tilde{X}\right)+\left.\delta \tilde{X}\left(g^{-1} \partial_{1} \tilde{X}-\frac{1}{2} \partial_{0} X\right)\right|_{\partial \Sigma}=0
$$

It looks like we have four options for the boundary conditions. However, $O(D, D)$ covariance excludes two of them and we are only left with two equally good boundary conditions

$$
\begin{gathered}
\left.\delta X\right|_{\partial \Sigma}=\left.\partial_{0} X\right|_{\partial \Sigma}=0 \\
g^{-1} \partial_{1} \tilde{X}-\left.\frac{1}{2} \partial_{0} X\right|_{\partial \Sigma}=\left.0 \Rightarrow \partial_{1} \tilde{X}\right|_{\partial \Sigma}=0
\end{gathered}
$$

or

$$
\begin{gathered}
\left.\delta \tilde{X}\right|_{\partial \Sigma}=\left.\partial_{0} \tilde{X}\right|_{\partial \Sigma}=0 \\
g \partial_{1} X-\left.\frac{1}{2} \partial_{0} \tilde{X}\right|_{\partial \Sigma}=\left.0 \Rightarrow \partial_{1} X\right|_{\partial \Sigma}=0 .
\end{gathered}
$$

Apparently, these two sets of boundary conditions agree with T-duality of open strings: if one boundary condition is Neumann, its T-dual boundary condition is Dirichlet. Since we eventually want to compare with the results in [1], hereafter we will select the second boundary condition (3.11). After applying (3.4) on the boundary, one gets $f_{1}(\tau)=0$. On the other hand, we can make a shift $X \rightarrow X-\int d \tau f_{2}(\tau)$ which does not affect the boundary conditions, EOM and the action. We are therefore free to set $f_{2}(\tau)=0$. The decoupled EOM now can be casted into

$$
\begin{aligned}
\left(\partial_{1}^{2}-\partial_{0}{ }^{2}\right) X & =0 \\
\left.\partial_{1} X\right|_{\partial \Sigma} & =0
\end{aligned}
$$


and

$$
\begin{aligned}
\left(\partial_{1}{ }^{2}-\partial_{0}{ }^{2}\right) \tilde{X} & =0, \\
\left.\partial_{0} \tilde{X}\right|_{\partial \Sigma} & =0 .
\end{aligned}
$$

From now on, we work with Euclidean signature by setting $\tau \rightarrow-i \tau\left(\partial_{0} \rightarrow i \partial_{0}\right)$, and use complex coordinates: $\partial_{0}=\partial+\bar{\partial}$ and $\partial_{1}=i(\partial-\bar{\partial})$. It is easy to figure out the propagators

$$
\begin{aligned}
& \left\langle X^{i}(z, \bar{z}) X^{j}\left(z^{\prime}, \bar{z}^{\prime}\right)\right\rangle=-\alpha^{\prime}\left(g^{i j} \log \left|z-z^{\prime}\right|+g^{i j} \log \left|z-\bar{z}^{\prime}\right|\right) . \\
& \left\langle\tilde{X}_{i}(z, \bar{z}) \tilde{X}_{j}\left(z^{\prime}, \bar{z}^{\prime}\right)\right\rangle=-\alpha^{\prime}\left(g_{i j} \log \left|z-z^{\prime}\right|-g_{i j} \log \left|z-\bar{z}^{\prime}\right|\right) .
\end{aligned}
$$

From these two propagators, $X$ and $\tilde{X}$ are both commutative. Our next step is to calculate the mixed propagators $\left\langle\tilde{X}_{i}(z, \bar{z}) X^{j}\left(z^{\prime}, \bar{z}^{\prime}\right)\right\rangle$ and $\left\langle X^{i}(z, \bar{z}) \tilde{X}_{j}\left(z^{\prime}, \bar{z}^{\prime}\right)\right\rangle$. From eq. (3.4) and (3.5) with $f_{i}=0$, we have

$$
g_{i j} \partial_{1} X^{j}=i \partial_{0} \tilde{X}_{i}, \quad g^{i j} \partial_{1} \tilde{X}_{j}=i \partial_{0} X^{i} .
$$

Therefore, the propagators satisfy the following equations

$$
\begin{aligned}
& g_{\ell i}(\partial-\bar{\partial})\left\langle X^{i}(z, \bar{z}) \tilde{X}_{j}\left(z^{\prime}, \bar{z}^{\prime}\right)\right\rangle=(\partial+\bar{\partial})\left\langle\tilde{X}_{\ell}(z, \bar{z}) \tilde{X}_{j}\left(z^{\prime}, \bar{z}^{\prime}\right)\right\rangle, \\
& g^{\ell i}(\partial-\bar{\partial})\left\langle\tilde{X}_{i}(z, \bar{z}) X^{j}\left(z^{\prime}, \bar{z}^{\prime}\right)\right\rangle=(\partial+\bar{\partial})\left\langle X^{\ell}(z, \bar{z}) X^{j}\left(z^{\prime}, \bar{z}^{\prime}\right)\right\rangle,
\end{aligned}
$$

The solutions of these two equations are

$$
\begin{aligned}
& \left\langle X^{i}(z, \bar{z}) \tilde{X}_{j}\left(z^{\prime}, \bar{z}^{\prime}\right)\right\rangle=-\frac{\alpha^{\prime}}{2} g^{i k} g_{k j}\left(\log \frac{z-z^{\prime}}{\bar{z}-\bar{z}^{\prime}}-\log \frac{z-\bar{z}^{\prime}}{\bar{z}-z^{\prime}}\right), \\
& \left\langle\tilde{X}_{i}(z, \bar{z}) X^{j}\left(z^{\prime}, \bar{z}^{\prime}\right)\right\rangle=-\frac{\alpha^{\prime}}{2} g_{i k} g^{k j}\left(\log \frac{z-z^{\prime}}{\bar{z}-\bar{z}^{\prime}}+\log \frac{z-\bar{z}^{\prime}}{\bar{z}-z^{\prime}}\right) .
\end{aligned}
$$

It is easy to check that these propagators also satisfy the boundary conditions. Considering the propagators on the boundary $z=\tau$ and $z^{\prime}=\tau^{\prime}$, we can get the commutators

$$
\begin{aligned}
{\left[X^{i}(\tau), X^{j}\left(\tau^{\prime}\right)\right] } & =\left[\tilde{X}^{i}(\tau), \tilde{X}^{j}\left(\tau^{\prime}\right)\right]=0 \\
{\left[\tilde{X}_{i}(\tau), X^{j}(\tau)\right] } & =i 2 \pi \alpha^{\prime} \delta_{i}{ }^{j} .
\end{aligned}
$$

Therefore, we find that $X$ or $\tilde{X}$ alone is commutative, but they do not commute with each other. This striking feature comes from the requirements that the double sigma model is $O(D, D)$ invariant and can reduce back to the Polyakov action. However, this noncommutativity is hidden from the ordinary picture. Since the action is $O(D, D)$ invariant, we now go to a general phase frame by a pure coordinate transformation

$$
\Omega=\left(\begin{array}{cc}
1 & -B^{i j} \\
0 & 1
\end{array}\right)
$$

where $B^{i j}$ is an antisymmetric tensor. The generalized metric $h_{M N}$ is then rotated to

$$
H_{M N}=\Omega^{T} h_{M N} \Omega=\left(\begin{array}{cc}
g & -g B^{-1} \\
B^{-1} g & g^{-1}-B^{-1} g B^{-1}
\end{array}\right),
$$


accompanied by the coordinate transformation

$$
\begin{aligned}
X^{i \prime} & =X^{i}+B^{i j} \tilde{X}_{j}, \\
\tilde{X}_{j}^{\prime} & =\tilde{X}_{j} .
\end{aligned}
$$

It is straightforward to calculate the propagators in the new frame

$$
\begin{aligned}
&\left\langle X^{i \prime}(z, \bar{z}) X^{j \prime}\left(z^{\prime}, \bar{z}^{\prime}\right)\right\rangle \\
&=\left\langle X^{i}(z, \bar{z})+B^{i k} \tilde{X}_{k}(z, \bar{z}), X^{j}\left(z^{\prime}, \bar{z}^{\prime}\right)+B^{j \ell} \tilde{X}_{l}\left(z^{\prime}, \bar{z}^{\prime}\right)\right\rangle \\
&=\left\langle X^{i}(z, \bar{z}), X^{j}\left(z^{\prime}, \bar{z}^{\prime}\right)\right\rangle-\left\langle X^{i}(z, \bar{z}), \tilde{X}_{\ell}\left(z^{\prime}, \bar{z}^{\prime}\right)\right\rangle B^{\ell j} \\
&+B^{i k}\left\langle\tilde{X}_{k}(z, \bar{z}), X^{j}\left(z^{\prime}, \bar{z}^{\prime}\right)\right\rangle-B^{i k}\left\langle\tilde{X}_{k}(z, \bar{z}), \tilde{X}_{\ell}\left(z^{\prime}, \bar{z}^{\prime}\right)\right\rangle B^{l j} \\
&=-\alpha^{\prime}\left[\left(g^{i j}-B^{i k} g_{k \ell} B^{\ell j}\right) \log \left|z-z^{\prime}\right|+\left(g^{i j}+B^{i k} g_{k \ell} B^{\ell j}\right) \log \left|z-\bar{z}^{\prime}\right|+B^{i j}\left(\log \frac{z-\bar{z}^{\prime}}{\bar{z}-z^{\prime}}\right)\right] .
\end{aligned}
$$

We see that the non-commutativity becomes visible in the last term. It is of importance to note that this propagator natively uses the open string metric and parameters. That is why it looks different from the propagator in [1] where the propagator was explicitly expressed with the closed string metric and parameters. Therefore, in order to make comparison, we need to rotate the parameters back to the closed string parameters. Rotating $H_{M N}$ back to the closed string parameters $\hat{g}$ and $\hat{B}$ is achieved by the transformation

$$
\eta\left(\begin{array}{cc}
\hat{g}^{-1} & -\hat{g}^{-1} \hat{B} \\
\hat{B} \hat{g}^{-1} & \hat{g}-\hat{B} \hat{g}^{-1} \hat{B}
\end{array}\right) \eta=\left(\begin{array}{cc}
\hat{g}-\hat{B} \hat{g}^{-1} \hat{B} & \hat{B} \hat{g}^{-1} \\
-\hat{g}^{-1} \hat{B} & \hat{g}^{-1}
\end{array}\right)=\left(\begin{array}{cc}
g & -g B^{-1} \\
B^{-1} g g^{-1}-B^{-1} g B^{-1}
\end{array}\right) .
$$

It gives us the following relations

$$
\begin{aligned}
g_{i j} & =\left(\hat{g}-\hat{B} \hat{g}^{-1} \hat{B}\right)_{i j}, \\
B^{i j} & =-\left(\frac{1}{\hat{g}+\hat{B}} \hat{B} \frac{1}{\hat{g}-\hat{B}}\right)^{i j}, \\
\hat{g}^{i j} & =\left(g^{-1}-B^{-1} g B^{-1}\right)^{i j} \\
\hat{B}^{i j} & =\left(B^{-1}-g^{-1} B g^{-1}\right)^{i j}
\end{aligned}
$$

which are precisely the transformations between closed and open parameters. Therefore, the propagator (3.25) can be rewritten with the closed string (hat) parameters as

$$
\begin{aligned}
& \left\langle X^{i \prime}(z, \bar{z}) X^{j \prime}\left(z^{\prime}, \bar{z}^{\prime}\right)\right\rangle=-\alpha^{\prime}\left[\hat{g}^{i j} \log \left|z-z^{\prime}\right|-\hat{g}^{i j} \log \left|z-\bar{z}^{\prime}\right|\right. \\
& \left.\quad+\left(\frac{1}{\hat{g}+\hat{B}} \hat{g} \frac{1}{\hat{g}-\hat{B}}\right)^{i j} \log \left|z-\bar{z}^{\prime}\right|^{2}+\left(-\frac{1}{\hat{g}+\hat{B}} \hat{B} \frac{1}{\hat{g}-\hat{B}}\right)^{i j}\left(\log \frac{z-\bar{z}^{\prime}}{\bar{z}-z^{\prime}}\right)\right],
\end{aligned}
$$

which is completely identical to the Seiberg-Witten result in [1]. It is of help to give the other three propagators with the closed string (hat) parameters

$$
\left\langle\tilde{X}_{i}^{\prime}(z, \bar{z}) \tilde{X}_{j}^{\prime}\left(z^{\prime}, \bar{z}^{\prime}\right)\right\rangle=-\alpha^{\prime}\left[\left(\hat{g}-\hat{B} \hat{g}^{-1} \hat{B}\right)_{i j} \log \left|z-z^{\prime}\right|-\left(\hat{g}-\hat{B} \hat{g}^{-1} \hat{B}\right)_{i j} \log \left|z-\bar{z}^{\prime}\right|\right],
$$




$$
\begin{aligned}
\left\langle X^{i \prime}(z, \bar{z}) \tilde{X}_{j}^{\prime}\left(z^{\prime}, \bar{z}^{\prime}\right)\right\rangle= & -\frac{\alpha^{\prime}}{2} \delta_{j}^{i}\left(\log \frac{z-z^{\prime}}{\bar{z}-\bar{z}^{\prime}}-\log \frac{z-\bar{z}^{\prime}}{\bar{z}-z^{\prime}}\right) \\
& -\alpha^{\prime}\left(\frac{1}{\hat{g}+\hat{B}} \hat{B} \frac{1}{\hat{g}-\hat{B}}\right)_{j}^{i}\left(\log \left|z-z^{\prime}\right|-\log \left|z-\bar{z}^{\prime}\right|\right), \\
\left\langle\tilde{X}_{i}^{\prime}(z, \bar{z}) X^{j^{\prime}}\left(z^{\prime}, \bar{z}^{\prime}\right)\right\rangle= & -\frac{\alpha^{\prime}}{2} \delta_{i}^{j}\left(\log \frac{z-z^{\prime}}{\bar{z}-\bar{z}^{\prime}}+\log \frac{z-\bar{z}^{\prime}}{\bar{z}-z^{\prime}}\right) \\
& +\alpha^{\prime}\left(\frac{1}{\hat{g}+\hat{B}} \hat{B} \frac{1}{\hat{g}-\hat{B}}\right)_{i}^{j}\left(\log \left|z-z^{\prime}\right|-\log \left|z-\bar{z}^{\prime}\right|\right) .
\end{aligned}
$$

We can easily see the commutators

$$
\begin{aligned}
{\left[X^{i \prime}(\tau), X^{j \prime}(\tau)\right] } & =i \pi \alpha^{\prime}\left(-\frac{1}{\hat{g}+\hat{B}} \hat{B} \frac{1}{\hat{g}-\hat{B}}\right)^{i j} \\
{\left[\tilde{X}_{i}^{\prime}(\tau), \tilde{X}_{j}^{\prime}(\tau)\right] } & =0 \\
{\left[\tilde{X}_{i}^{\prime}(\tau), X^{j \prime}(\tau)\right] } & =i 2 \pi \alpha^{\prime} \delta_{i}{ }^{j} .
\end{aligned}
$$

To see how the Seiberg-Witten map arises, we eliminate $\tilde{X}$ from the double sigma action (3.1) in general frames. Using the EOM of $\tilde{X}$ and the boundary condition $\left.\delta \tilde{X}\right|_{\partial \Sigma}=0$, after a bit calculation, we get the action with Euclidean signature

$$
\begin{aligned}
S=\frac{1}{2} \int_{\Sigma} \partial_{a} X^{\prime} & \left(\frac{1}{g^{-1}-B^{-1}} g^{-1} \frac{1}{g^{-1}+B^{-1}}\right) \partial^{a} X^{\prime} \\
& -2 i \partial_{0} X\left(-\frac{1}{g^{-1}-B^{-1}} B^{-1} \frac{1}{g^{-1}-B^{-1}}\right) \partial_{1} X,
\end{aligned}
$$

which confirms eq. (3.26). It is readily to obtain the DBI of this action

$$
\begin{aligned}
S_{D B I} & =\frac{1}{g_{s}} \int d^{D} x \sqrt{\operatorname{det}\left(\frac{1}{g^{-1}+B^{-1}}+F\right)} \\
& =\frac{1}{g_{s}} \int d^{D} x \sqrt{\hat{g}+\hat{B}+F} \\
& =\frac{1}{G_{s}} \int d^{D} x \sqrt{\operatorname{det}\left(g+F^{*}\right)},
\end{aligned}
$$

with

$$
F^{*}=\frac{1}{1+F B^{-1}} F, \quad G_{s} \equiv g_{s} \sqrt{\operatorname{det}\left(1+B^{-1} g\right)},
$$

where $F^{*}$ is the non-commutative gauge field strength and $F$ is the commutative one. It is identical to that of Seiberg-Witten, but the physical interpretation is now changed: $B^{-1}$ is an $O(D, D)$ group parameter. It is now clear that in the original work of Seiberg-Witten, only one set of coordinates is visible. There is no non-commutativity at the very beginning when $\hat{B}=0$, since the mixed non-commutative propagators $\left\langle\tilde{X}^{i}(z, \bar{z}) X_{j}\left(z^{\prime}, \bar{z}^{\prime}\right)\right\rangle$ is hidden. Non-commutativity emerges upon turning on a boundary term. However, when we begin with the double sigma model, non-commutativity is an intrinsic property and one can freely transform a commutative sector to a non-commutative one or vice versa. The SeibergWitten map is really just a symmetry. More importantly, the metric or parameters in the 
double sigma model are natively determined to be open or closed parameters by the action itself upon imposing $O(D, D)$ covariant boundary conditions as demonstrated in eq. (3.32).

It is interesting to look at the propagator (3.29), which is commutative even if we have a non-vanishing $\hat{B}$ field. This propagator extends the results of Seiberg-Witten map. In an abstract way, we generalize the open-closed string relation from equations (3.26) and (3.28):

$$
\left(\begin{array}{cc}
\hat{g}-\hat{B} \hat{g}^{-1} \hat{B} & \hat{B} \hat{g}^{-1} \\
-\hat{g}^{-1} \hat{B} & \hat{g}^{-1}
\end{array}\right)=\left(\begin{array}{cc}
G & G \theta \\
-\theta G & G^{-1}-\theta G \theta
\end{array}\right)
$$

where we identify the open string metric $G \equiv g$ and $\theta \equiv B$. It gives a generalized map between open string variables $\left(G_{s}, G_{i j}, \theta_{i j}\right)$ and closed string variables $\left(g_{s}, \hat{g}_{i j}, \hat{B}_{i j}\right)$. When $\hat{B}=0$, we get

$$
\left(\begin{array}{cc}
\hat{g} & 0 \\
0 & \hat{g}^{-1}
\end{array}\right)=\left(\begin{array}{cc}
G & 0 \\
0 & G^{-1}
\end{array}\right), \quad G=g
$$

where two sides are all commutative. However, when $\hat{B} \neq 0$, we can also obtain commutativity through

$$
\left(\begin{array}{ll}
1 & 0 \\
\theta & 1
\end{array}\right)\left(\begin{array}{cc}
G & G \theta \\
-\theta G & G^{-1}-\theta G \theta
\end{array}\right)\left(\begin{array}{cc}
1 & -\theta \\
0 & 1
\end{array}\right)=\left(\begin{array}{cc}
G & 0 \\
0 & G^{-1}
\end{array}\right), \quad G=\hat{g}-\hat{B} \hat{g}^{-1} \hat{B}
$$

which corresponds to the propagator (3.29). Therefore, when $B \neq 0$, there also exists commutativity.

\section{The general descriptions of Seiberg-Witten map}

From eq. (3.33) and (3.27), the ${ }^{*}$ product of the non-commutative Yang-Mills theory $F^{*}$ is defined by a definite quantity: $B^{-1}=-(\hat{g}+\hat{B})^{-1} \hat{B}(\hat{g}-\hat{B})^{-1}$. In [1], Seiberg and Witten proposed that there exist more general descriptions of the map, with an arbitrary parameter $\theta$ but not just $B^{-1}$ and the $\theta$ dependence of the effective action is completely captured by replacing

$$
F^{*} \rightarrow F^{*}+\Phi
$$

where $\Phi$ is some two-form depending on $\hat{B}, \hat{g}$ and $\theta$, called the description parameter, determined by

$$
\begin{aligned}
\frac{1}{g+\Phi} & =-\theta+\frac{1}{\hat{g}+\hat{B}} \\
G_{s} & =g_{s}\left(\frac{\operatorname{det}(g+\Phi)}{\operatorname{det}(\hat{g}+\hat{B})}\right)^{\frac{1}{2}}=g_{s} \frac{1}{\operatorname{det}\left[\left(\frac{1}{\hat{g}+\hat{B}}-\theta\right)(\hat{g}+\hat{B})\right]^{\frac{1}{2}}}
\end{aligned}
$$

with the non-commutative effective action

$$
\mathcal{L}_{D B I}^{*}=\frac{1}{G_{s}} \sqrt{\operatorname{det}\left(G+F^{*}+\Phi\right)}
$$


To see what we can get from $O(D, D)$ on general descriptions, we make a second rotation following that of (3.22)

$$
\Omega^{\prime}=\left(\begin{array}{cc}
1 & -B^{-1} \\
0 & 1
\end{array}\right)\left(\begin{array}{cc}
1 & 0 \\
-C & 1
\end{array}\right)=\left(\begin{array}{cc}
1+B^{-1} C & -B^{-1} \\
-C & 1
\end{array}\right)
$$

where $B$ and $C$ are two-forms. The generalized metric under this rotation is

$$
H_{M N}^{\prime}=\left(\begin{array}{cc}
g+C B^{-1} g+g B^{-1} C-C g^{-1} C+C B^{-1} g B^{-1} C & -g B^{-1}+C g^{-1}-C B^{-1} g B^{-1} \\
B^{-1} g-g^{-1} C+B^{-1} g B^{-1} C & g^{-1}-B^{-1} g B^{-1}
\end{array}\right) \text {. }
$$

The corresponding coordinate transformations are

$$
\begin{aligned}
& X^{\prime}=X+B^{-1} \tilde{X} \\
& \tilde{X}^{\prime}=C X+\left(1+C B^{-1}\right) \tilde{X}
\end{aligned}
$$

It is easy to imagine that with these coordinate transformations, both $X^{\prime}$ and $\tilde{X}^{\prime}$ are non-commutative. Applying (3.26), we identify

$$
\begin{aligned}
\hat{g}^{-1} & =g^{-1}-B^{-1} g B^{-1} \\
\hat{B} & =C-\frac{1}{g^{-1}+B^{-1}} B^{-1} \frac{1}{g^{-1}-B^{-1}} \\
\hat{g}+\hat{B} & =C+\frac{1}{g^{-1}+B^{-1}}
\end{aligned}
$$

Then we have the commutative DBI

$$
\begin{aligned}
S_{D B I} & =\frac{1}{g_{s}} \int d^{D} x \sqrt{\operatorname{det}(\hat{g}+\hat{B}+F)} \\
& =\frac{1}{g_{s}} \int d^{D} x \sqrt{\operatorname{det}\left(\frac{1}{g^{-1}+B^{-1}}+F+C\right)}
\end{aligned}
$$

After a bit calculations, one can prove that the non-commutative description is

$$
S_{D B I}=\frac{1}{G_{s}} \int d^{D} x \sqrt{\operatorname{det}\left(g+F^{*}+\Phi\right)},
$$

with $G_{s}$ defined by (4.3) and the constraint for $\Phi$ and $\theta$

$$
\frac{1}{g+\Phi}+\theta=\frac{1}{C+\frac{1}{g^{-1}+B^{-1}}}=\frac{1}{\hat{g}+\hat{B}}
$$

and

$$
F^{*}=\frac{1}{1+F \theta} F
$$

A remarkable observation is that from (4.7), for fixed closed string $\hat{g}$ and $\hat{B}$, the open string parameters $g$ and $B$ are still free to vary provided $C$ varying accordingly. Referring to (4.9), $\Phi$ is unnecessary to keep $\theta$ varying and we can therefore set $\Phi=0$ in (4.8) 
and (4.9). Therefore, $C$ actually plays the role of the description parameter and in the non-commutative DBI, its effect is incorporated into the variation of the open string metric $g$. This tells us that one does not need to introduce an independent field and the $O(D, D)$ symmetry already has this ingredient.

In summary, we start from the double sigma model and found that for open strings, the theory is intrinsically non-commutative. In the simplest phase state, the ordinarily visible sector is commutative and the non-commutativity is hidden. In a general frame, the noncommutativity arises from $O(D, D)$ rotations. The visible sector completely agrees with previous results. We showed that the parameters of the double sigma model are determined to be open or closed by the boundary conditions. The open and closed parameters are related by an $O(D, D)$ transformation. We further exhibited that as $\hat{B} \neq 0$, besides the noncommutative theory, there is another commutative one. Our results demonstrated that the Seiberg-Witten map is actually a subset of $O(D, D)$ symmetry. Finally, we explored double rotations and found that the general descriptions of Seiberg-Witten map is also naturally derived by a group parameter. In this scenario, all the sectors are non-commutative. It is quite interesting that there are a lot of similarities between our results and the noncommutativity from closed strings [33-35]. These similarities cannot be accident and need more explorations in the future works.

\section{Acknowledgments}

We would like to acknowledge illuminating discussions with Zheng Sun, Yan He and Bo Feng. This work is supported in part by the NSFC (Grant No. 11175039 and 11375121 ) and SiChuan Province Science Foundation for Youths (Grant No. 2012JQ0039).

Open Access. This article is distributed under the terms of the Creative Commons Attribution License (CC-BY 4.0), which permits any use, distribution and reproduction in any medium, provided the original author(s) and source are credited.

\section{References}

[1] N. Seiberg and E. Witten, String theory and noncommutative geometry, JHEP 09 (1999) 032 [hep-th/9908142] [INSPIRE].

[2] A. Connes, M.R. Douglas and A.S. Schwarz, Noncommutative geometry and matrix theory: compactification on tori, JHEP 02 (1998) 003 [hep-th/9711162] [INSPIRE].

[3] C.-S. Chu and P.-M. Ho, Noncommutative open string and D-brane, Nucl. Phys. B 550 (1999) 151 [hep-th/9812219] [INSPIRE].

[4] C.-S. Chu and P.-M. Ho, Constrained quantization of open string in background B field and noncommutative D-brane, Nucl. Phys. B 568 (2000) 447 [hep-th/9906192] [INSPIRE].

[5] J. Maharana and S.S. Pal, Noncommutative open string, D-brane and duality, Phys. Lett. B 488 (2000) 410 [hep-th/0005113] [INSPIRE].

[6] A.A. Tseytlin, Duality symmetric formulation of string world sheet dynamics, Phys. Lett. B 242 (1990) 163 [INSPIRE]. 
[7] A.A. Tseytlin, Duality symmetric closed string theory and interacting chiral scalars, Nucl. Phys. B 350 (1991) 395 [inSPIRE].

[8] J. Maharana and J.H. Schwarz, Noncompact symmetries in string theory, Nucl. Phys. B 390 (1993) 3 [hep-th/9207016] [InSPIRE].

[9] J.H. Schwarz and A. Sen, Duality symmetric actions, Nucl. Phys. B 411 (1994) 35 [hep-th/9304154] [INSPIRE].

[10] W. Siegel, Two vierbein formalism for string inspired axionic gravity, Phys. Rev. D 47 (1993) 5453 [hep-th/9302036] [INSPIRE].

[11] W. Siegel, Superspace duality in low-energy superstrings, Phys. Rev. D 48 (1993) 2826 [hep-th/9305073] [INSPIRE].

[12] W. Siegel, Manifest duality in low-energy superstrings, in Proceedings, Strings '93, Berkeley CA U.S.A. (1993), pg. 353, and State U. New York Stony Brook - ITP-SB-93-050, U.S.A. (1993) [hep-th/9308133] [INSPIRE].

[13] C. Hull and B. Zwiebach, Double field theory, JHEP 09 (2009) 099 [arXiv:0904.4664] [INSPIRE].

[14] O. Hohm, C. Hull and B. Zwiebach, Background independent action for double field theory, JHEP 07 (2010) 016 [arXiv: 1003.5027] [INSPIRE].

[15] O. Hohm, C. Hull and B. Zwiebach, Generalized metric formulation of double field theory, JHEP 08 (2010) 008 [arXiv: 1006.4823] [InSPIRE].

[16] M.J. Duff, Duality rotations in string theory, Nucl. Phys. B 335 (1990) 610 [InSPIRE].

[17] D.S. Berman, N.B. Copland and D.C. Thompson, Background field equations for the duality symmetric string, Nucl. Phys. B 791 (2008) 175 [arXiv:0708.2267] [INSPIRE].

[18] D.S. Berman and D.C. Thompson, Duality symmetric strings, dilatons and $O(D, D)$ effective actions, Phys. Lett. B 662 (2008) 279 [arXiv:0712.1121] [INSPIRE].

[19] N.B. Copland, Connecting T-duality invariant theories, Nucl. Phys. B 854 (2012) 575 [arXiv:1106.1888] [INSPIRE].

[20] N.B. Copland, A double $\sigma$-model for double field theory, JHEP 04 (2012) 044 [arXiv: 1111.1828] [INSPIRE].

[21] D.S. Berman and D.C. Thompson, Duality symmetric string and M-theory, Phys. Rept. 566 (2014) 1 [arXiv:1306.2643] [InSPIRE].

[22] K. Lee and J.-H. Park, Covariant action for a string in "doubled yet gauged" spacetime, Nucl. Phys. B 880 (2014) 134 [arXiv:1307.8377] [INSPIRE].

[23] C.-T. Ma, One-loop $\beta$ function of the double $\sigma$-model with constant background, arXiv:1412.1919 [INSPIRE].

[24] E.S. Fradkin and A.A. Tseytlin, Nonlinear electrodynamics from quantized strings, Phys. Lett. B 163 (1985) 123 [INSPIRE].

[25] C.G. Callan Jr., C. Lovelace, C.R. Nappi and S.A. Yost, String loop corrections to $\beta$-functions, Nucl. Phys. B 288 (1987) 525 [INSPIRE].

[26] A. Abouelsaood, C.G. Callan Jr., C.R. Nappi and S.A. Yost, Open strings in background gauge fields, Nucl. Phys. B 280 (1987) 599 [INSPIRE]. 
[27] S. Mukhi, Noncommutativity in string theory, talk given at PiTP, IAS, Princeton U.S.A. July 1-12 2002.

[28] C.M. Hull, A geometry for non-geometric string backgrounds, JHEP 10 (2005) 065 [hep-th/0406102] [INSPIRE].

[29] C.M. Hull, Doubled geometry and T-folds, JHEP 07 (2007) 080 [hep-th/0605149] [INSPIRE].

[30] H.S. Yang, Exact Seiberg-Witten map and induced gravity from noncommutativity, Mod. Phys. Lett. A 21 (2006) 2637 [hep-th/0402002] [INSPIRE].

[31] H.S. Yang, Emergent gravity from noncommutative spacetime, Int. J. Mod. Phys. A 24 (2009) 4473 [hep-th/0611174] [inSPIRE].

[32] H.S. Yang, Highly effective action from large- $N$ gauge fields, Phys. Rev. D 90 (2014) 086006 [arXiv: 1402.5134] [INSPIRE].

[33] D. Andriot, M. Larfors, D. Lüst and P. Patalong, A ten-dimensional action for non-geometric fluxes, JHEP 09 (2011) 134 [arXiv:1106.4015] [INSPIRE].

[34] D. Andriot, M. Larfors, D. Lüst and P. Patalong, (Non-)commutative closed string on T-dual toroidal backgrounds, JHEP 06 (2013) 021 [arXiv:1211.6437] [INSPIRE].

[35] C.D.A. Blair, Non-commutativity and non-associativity of the doubled string in non-geometric backgrounds, arXiv:1405.2283 [INSPIRE]. 